\title{
USO DO ÍNDICE DE ESTADO TRÓFICO E ANÁLISE RÁPIDA DA COMUNIDADE DE MACROINVERTEBRADOS COMO INDICADORES DA QUALIDADE AMBIENTAL DAS ÁGUAS NA BACIA DO RIO JUNDIAÍ-MIRIM - SP - BR
}

\author{
BEGHELLI, F.G.S'1.; CARVALHO, M.E.K ${ }^{1}$.; PECHE FILHO, A ${ }^{12}$.; MACHADO, F.H ${ }^{1}$.; MOSCHINI-CARLOS \\ $V^{1}$.; POMPÊO, M.L.M ${ }^{13}$;; RIBEIRO, A.I'.; MEDEIROS, G.A ${ }^{1}$. \\ 1 Universidade Estadual Paulista "Júlio de Mesquita Filho" - UNESP, Sorocaba, SP, Brasil. \\ 2 Instituto Agronômico de Campinas - IAC, Jundiaí, SP, Brasil. \\ 3 Departamento de Ecologia, IB, Universidade de São Paulo - USP, São Paulo, SP, Brasil. \\ *Corresponding author: fred_sb@hotmail.com
}

\begin{abstract}
Beghelli, F.G.S.; Carvalho, M.E.K.; Peche Filho, A.; Machado, F.H.; Moschini-Carlos, V.; Pompêo, M.L.M.; Ribeiro, A.I.; Medeiros, G.A. 2014. Uso do índice de estado trófico e análise rápida da comunidade de macroinvertebrados como indicadores da qualidade ambiental das águas na Bacia do Rio Jundiaí-Mirim - SP - BR. Braz. J. Aquat. Sci. Technol. 19(1):13-22. eISSN 1983-9057. DOI: 10.14210/bjast.v19n1.3p13-22 The Jundiaí-Mirim watershed supplies the Jundiaí city and it is located between two metropolitan regions: the Campinas metropolitan region and the São Paulo metropolitan region which are the two most populous regions in São Paulo state. In this region some problems such as hydric resources scarcity, as well the water quality; conflicts between the water users and hydric pollution are part of the reality faced by the management agencies and government. The trophic state index (TSI) allows the water bodies classification according to the nutrients entrance into the system. That contribution artificially stimulates the algae and macrophytes proliferation and may causes a number of problems which includes: toxic algae proliferation, fish kills and disagreeable odor. The benthic macroinvertebrates are one of the most widely and recognized bioindicators in the aquatic environments reacting to impacts in water column, sediment and margins. The objective of the present research was to assess the environmental quality of the Jundiaí Mirim basin based on the trophic state index and the benthic macroinvertebrates community. Samplings were performed for TSI calculation and benthic macroinvertebrates rapid analysis at four samplings stations along the Jundiaí-Mirim river basin (Jundiaí, SP, Brazil). The data obtained reveals a worrying situation of the basin which is suffering a eutrophication process resulting in a poor quality of its waters and sediments. Moreover, it was recorded the presence of the exotic mollusk Corbicula fluminea, one of the freshwater exotic species of main concern in the world. It is recommended the development of an action project comprising short, average and long term marks in order to promote the recovery of the environment and to avoid environmental, social and economic problems. A rapid analysis index using the benthic macroinvertebrates was proposed.
\end{abstract}

Keywords: bioindicator, water quality, rapid assessment protocols, Corbicula fluminea, benthic macroinvertebrates.

\section{INTRODUÇÃO}

Ambientes de águas continentais são essenciais à vida e bem estar da humanidade. A espécie humana depende destes ambientes para suprimento de água em quantidade e qualidade a fim de atender as suas necessidades biológicas, de lazer e econômicas (ONU, 1992).

Com o desenvolvimento da agricultura, das cidades e da industrialização estes recursos adquiriram importância em diferentes níveis e os ecossistemas aquáticos passaram então a sofrer mais impactos com a intensificação progressiva de seus usos múltiplos como dessedentação, pecuária, irrigação de culturas, despejo de efluentes, utilização em processos industriais, pesca, transporte, dentre outros. Para que a água seja mantida em quantidade e qualidade suficientes a estas necessidades, a manutenção dos processos ecológicos é fundamental (Baron et al., 2003).
As atividades humanas têm ocasionado uma série de impactos sobre estes sistemas como supressão de mata ciliar (Dosskey et al., 2010), eutrofização das águas devido ao aporte excessivo de nutrientes provenientes principalmente de atividades agropecuárias (Monteagudo et al., 2012; Pedrazzi et al., 2013; Smith et al., 2013) e despejo de efluentes (Thevenon et al., 2011; Lawal et al., 2013), alterações do regime hídrico (Costigan \& Daniels, 2012), poluição por metais pesados (Thevenon et al., 2011; Medeiros et al., 2013) e agrotóxicos (Moreira et al., 2012), introdução de espécies exóticas (Sousa et al., 2008) e assoreamento (Melo et al., 2004).

Tais impactos fragilizam e alteram as condições do meio aquático, descaracterizando-o, podendo atingir níveis de perdas ambientais que comprometem a fonte de recursos para manutenção da vida, abastecimento ou mesmo recreação como ocorre com o rio Tietê, na região da grande São Paulo (Rodgher et al., 2005; Zanata et al., 2008; Cunha et al., 2011). 
Estudos têm auxiliado na melhor compreensão dos efeitos impactantes de atividades humanas nestes ambientes e fornecido ferramentas e informações para sua gestão, conservação e mesmo recuperação (Stewart et al., 2011; Floehr et al., 2013).

Indicadores físicos, químicos e biológicos têm sido utilizados para diagnosticar a qualidade da água e fomentar políticas públicas de recuperação e preservação dos recursos hídricos (US-EPA, 2003; Caldas \& Sanches Filho, 2013; CETESB, 2014).

Nesse contexto macroinvertebrados bentônicos são organismos sésseis ou com mobilidade reduzida que vivem no sedimento ou próximo a ele. São majoritariamente detritívoros recebendo alimento, virtualmente, de todos os demais níveis tróficos, bem como do sistema terrestre adjacente e ambientes aquáticos à montante, atuando inclusive na recuperação de nutrientes e energia (Li et al., 2010).

Trata-se de uma comunidade com características desejáveis a bioindicadores: diversidade de organismos, ciclo de vida relativamente longo, estão presentes em praticamente qualquer corpo d'água e respondem a impactos variados. Por estes motivos, além de viverem no fundo, onde ocorre o acúmulo de poluentes, estes organismos muitas vezes têm sido considerados por pesquisadores e agências ambientais como um dos melhores indicadores de qualidade de um corpo d'água (Mandaville, 2002; US-EPA, 2012).

A eutrofização consiste no enriquecimento de um corpo d'água sendo a forma artificial um processo prejudicial ao sistema e que ocorre em um curto espaço de tempo. Dentre outros, este processo tende a comprometer a qualidade da água e do ecossistema por levar às seguintes consequencias: redução nas concentrações de oxigênio dissolvido, provocando a morte de muitos organismos, incluindo peixes; proliferação da biomassa fitoplanctônica e aumento das populações de cianobactérias potencialmente tóxicas; assoreamento; proliferação de macrófitas aquáticas que podem resultar em problemas como entupimento de tubulações, retenção de partículas e danos a turbinas geradoras de energia, além de mau cheiro (Tundisi \& Matsumura-Tundisi, 2008).

Assim sendo, este trabalho tem por objetivos realizar uma análise diagnóstica das condições da bacia do rio Jundiaí-Mirim, um importante manancial localizado entre as regiões metropolitanas de São Paulo e de Campinas baseado na proposta de uma análise rápida da comunidade de macroinvertebrados bentônicos associada ao IET.

\section{Área de Estudo}

A microbacia do rio Jundiaí-Mirim insere-se na Unidade de Gerenciamento de Recursos Hídricos 5 (UGRHI-5) que abrange as bacias hidrográficas dos rios Jundiaí, Piracicaba e Capivari. A microbacia do rio Jundiaí-Mirim, estende-se pelos municípios de Jundiaí (55\% da área da bacia), Jarinú (37\%) e Campo Limpo Paulista (8\%), ocupando uma área de $1175.105 \mathrm{~m}^{2}$ (Figura 1). Ao final da área de drenagem dessa microbacia existem dois reservatórios artificiais responsáveis pelo fornecimento de $97 \%$ da água consumida pelo município de Jundiaí (Moraes, 2003).

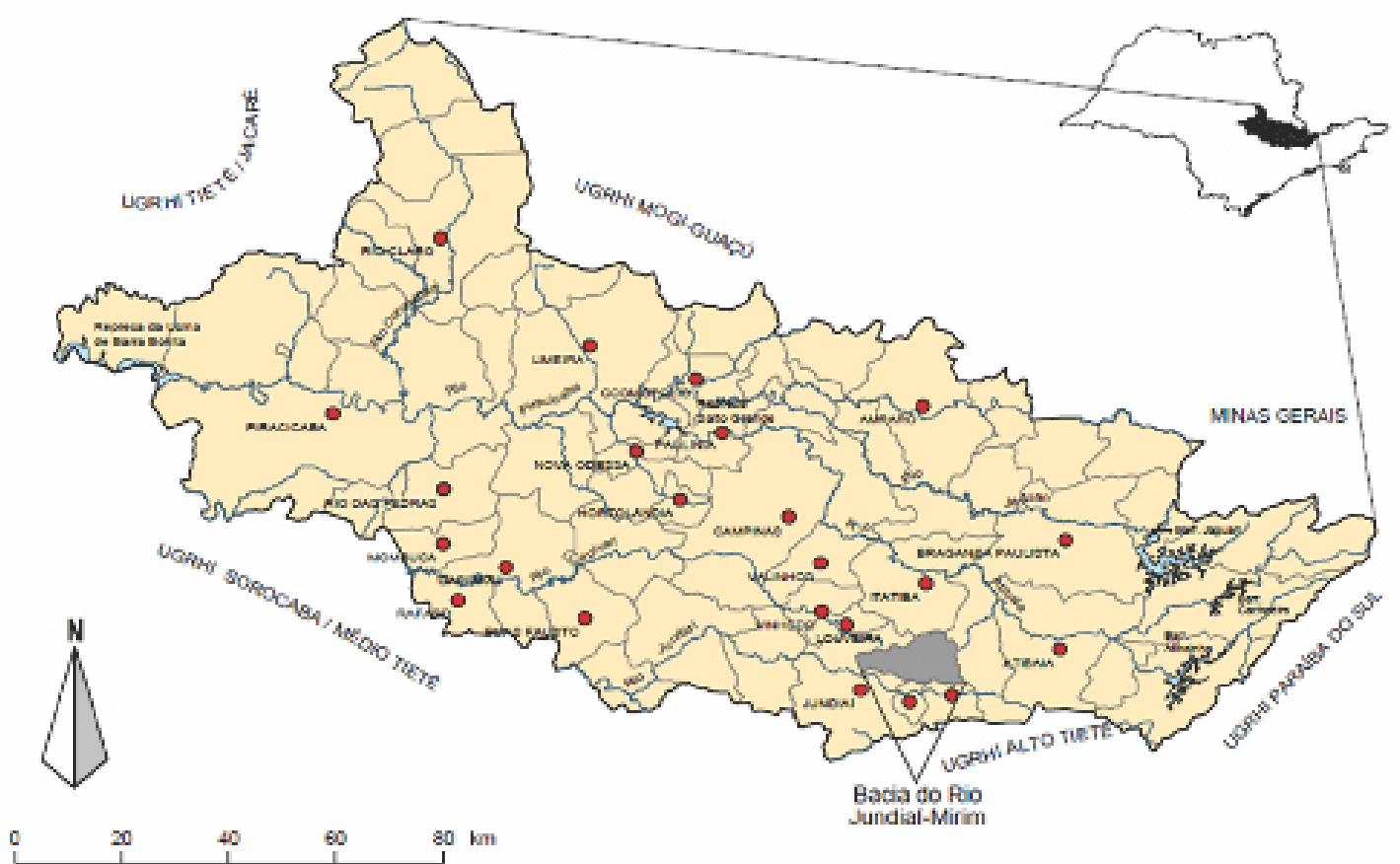

Figura 1 - Localização da microbacia do rio Jundiaí-Mirim. Fonte: Moraes (2003). 
Com base em dados da Fundação Sistema Estadual de Análise de Dados (SEADE, 2014) e do Instituto Brasileiro de Geografia e Estatística (IBGE, 2010), dos municípios que compõem a bacia, Jundiaí é o que apresenta o maior Produto Interno Bruto PIB e PIB per capta (ano de referência: 2010). O PIB deste município é de $\mathrm{R} \$ 20.124,60$ milhões $(1,6 \%$ do PIB do Estado), com PIB per capta de R $\$ 54.433,47$. Campo Limpo Paulista possui um PIB de R\$ 975,78 milhões ( $0,08 \%$ do PIB do Estado), com PIB per capta de $R \$ 13.189,65$. Jarinu possui um PIB de $R \$ 426,67$ milhões (0,03\% do PIB do Estado), com PIB per capta de $R \$ 17.942,46$.

Segundo Freitas (2012), dentre os impactos que a bacia sofre, destacam-se: a presença e expansão de loteamentos, a urbanização crescente da região e as atividades de mineração desenvolvidas.

Além destas, outras pressões também são verificadas como supressão de mata ciliar, utilização da área de APP para cultivos agrícolas e criação de animais bem como para plantio de Eucalyptus sp de forma que apenas $41 \%$ da área de APP - que deveria abrigar mata nativa para manutenção da qualidade das águas - realmente o fazem (Moraes, 2003).

\section{MATERIAIS E MÉTODOS}

\section{Amostragem}

Com o objetivo de se realizar uma análise expedita, de diagnóstico, foram coletadas amostras em um único esforço amostral em 02 de setembro de 2013 para análise da comunidade de macroinvertebrados bentônicos bem como para leitura das concentrações de fósforo e clorofila a em quatro pontos amostrais (tabela 1).

\section{Macroinvertebrados bentônicos}

As amostras de macroinvertebrados bentônicos foram realizadas com a utilização de uma draga do tipo Van-Veen (área $=405 \mathrm{~cm}^{2}$ ) em duplicata amostras cumulativas - para os pontos A, B, C e D. No campo, estas amostras foram lavadas sobre peneira com 0,2 $\mathrm{mm}$ de abertura de malha e então foi adicionado formaldeído às amostras de tal forma que sua concentração final fosse de $4 \%$.

Foi aplicado um protocolo para monitoramento rápido da seguinte forma: as amostras foram homogeneizadas triadas de forma aleatória até a obtenção de 100 indivíduos. Quando este limite não foi atingido, a amostra foi triada por completo (Mandaville, 2002).

Lâminas descartáveis foram montadas com água para a identificação sob microscópio óptico dos seguintes grupos: Chironominae, Tanytarsini, Tanypodinae, Chironomini vermelhos, Tubificinae e Naidinae. Foram utilizadas as seguintes chaves de identificação: Trivinho-Strixino (2011); Marchese (2009); Leal et al. (2013).

$\mathrm{Na}$ análise foi aplicado um índice multimétrico variando de 0-1 a partir da média aritmética de um conjunto de índices e métricas modificados tomando como base estudos anteriores que já caracterizaram os grupos utilizados como sensíveis a variações ambientais provocadas por interferências antrópicas (tabela 2).

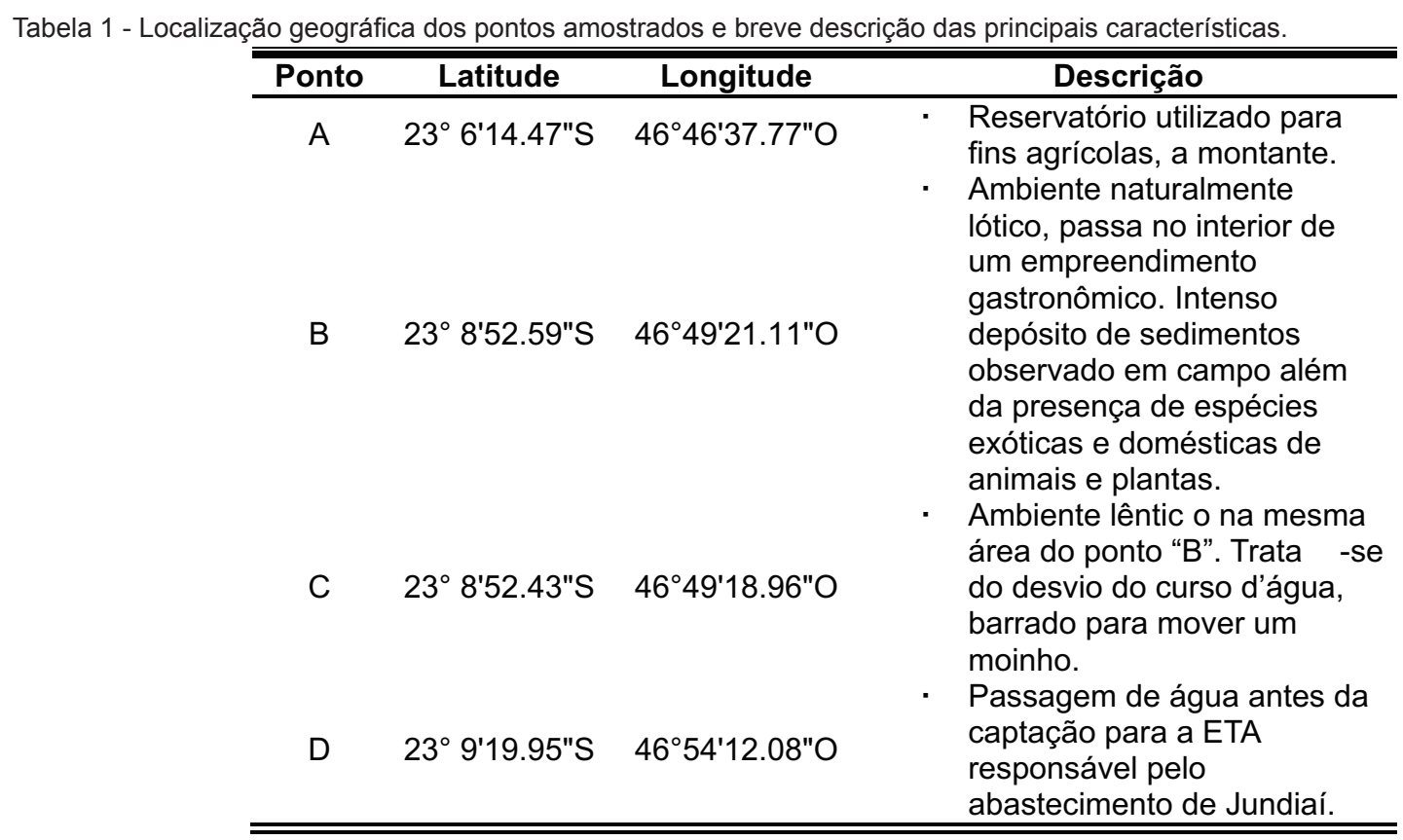


Desta forma, os índices e métricas utilizados para a composição do índice multimétrico final, baseiam-se em proporções entre organismos tolerantes e sensíveis, refletindo impactos como baixas concentrações de oxigênio, supressão de mata ripária e eutrofização. Também é utilizado o índice "family biotic index" (modificado em uma faixa de 0 a 1 ) que se baseia em escores de tolerância à poluição orgânica (Hilsenhoff, 1988).

Ademais, os grupos escolhidos para comporem o índice são de distribuição cosmopolita. Táxons de distribuição mais específica não foram incluídos. Considerando-se os grandes impactos que espécies exóticas podem causar ao invadirem um ambiente (Knowler, 2005), a proporção destes elementos também foi considerada.

Por fim, foi utilizada uma diversidade aparente (já que não houve quantificação de grupos taxonômicos) partindo do princípio que um ambiente impactado é, em geral, mais simplificado em termos de diversidade do que um ambiente não impactado. Este índice multimétrico classifica o corpo d'água em sete categorias conforme tabela 3 .

Foram coletadas amostras de água para determinação das concentrações de fósforo total e clorofila a para cálculo do índice de estado trófico de Carlson (1977) modificado por Lamparelli (2004), sendo adotado o índice para ambientes lóticos nos pontos $B$ e $D$ e lênticos para os pontos $A$ e $B$.

As determinações das concentrações de fósforo e clorofila a foram obtidas a partir de amostras de água subsuperficial. Para determinação de clorofila a, foi filtrado $1 \mathrm{~L}$ da amostra com filtro de microfibra de vidro (Whatman GF/C). Após a filtragem, o material foi macerado com adição de acetona $10 \%$ alcalinizada com $\mathrm{NH}_{4} \mathrm{OH}(\mathrm{pH} 8,0)$ para extração da clorofila. As amostras foram então mantidas no escuro, em geladeira, por $12 \mathrm{~h}$ e então centrifugadas a $3000 \mathrm{rpm}$ por cinco minutos. A leitura foi realizada nos comprimen- tos de onda de 665 e $750 \mathrm{~nm}$. A seguir o material foi acidificado com $\mathrm{HCl}$ para $\mathrm{pH} \mathrm{3,0} \mathrm{e} \mathrm{foi} \mathrm{realizada} \mathrm{nova}$ leitura. Os cálculos foram feitos segundo Wetzel \& Likens, (1991).

As concentrações de fósforo total foram determinadas pelo método de molibdovanadato com digestão por persulfato ácido a partir de amostras não filtradas com leitura em espectrofotômetro $\mathrm{HACH}$ DR-3900, conforme descrito pelo método 10127 do manual de procedimentos do espectrofotômetro $\mathrm{HACH}$. Uma regressão linear foi realizada para verificar se as concentrações de clorofila a realmente estariam variando em função do aporte de fósforo no sistema.

\section{RESULTADOS}

\section{Clorofila a, fósforo e o índice de estado trófico}

Os dados obtidos indicam que os níveis de fósforo na água estão relacionados à biomassa fitoplanctônica (tabela 4 e figura 2). Com relação ao IET, os pontos foram considerados de meso a supereutróficos conforme apresentado na tabela 5 .

A densidade de organismos bentônicos variou de 37 a >>1235 ind. $\mathrm{m}^{-2}$ sendo este último valor bem inferior ao real uma vez que o ponto $B$ não teve a totalidade da amostra contada $(n>100)$. Neste ponto foram analisados 100 organismos (1235 ind. $\mathrm{m}^{-2}$ ) em, aproximadamente, um quinto da amostra total e, portanto, estima-se uma densidade máxima superior a 6000 ind. $\mathrm{m}^{-2}$ (figura 3 ).

A composição das comunidades de cada ponto amostral conforme o grupo considerado está apresentada na figura 4 . Houve predomínio dos grupos associados às condições de maior impacto (Tubificinae, Corbicula fluminea e quironomídeos vermelhos).

Tabela 2 - Métricas e índices modificados utilizados para a composição do índice multimétrico desenvolvido para análise rápida da comunidade de macroinvertebrados bentônicos da bacia do rio Jundiaí-Mirim. Chr = Chironominae; Tub = Tubificinae; Exo = espécies exóticas; Pent. Verm.= Pentaneurini vermelhos; Tnt = Tanytarsinae; Chd = Chironomidae (inclui Tanytarsinae e demais Chironomidae); $\mathrm{AR}=$ abundância relativa.

\begin{tabular}{cc}
\hline \hline Índices / Métricas & Referências \\
\hline Diversidade de Simpson & Odum (1988) \\
1- Family Biotic Index & Mandaville (2002) \\
1- Proporção de organismos & Mandaville, (2002); Fusari \& Fonseca- \\
tolerantes (Tub+Chr verm + Exo + & Guessner (2006); Trivinho-Strixino \\
Pent. Verm) & $(2011)$ \\
Tnt/Chd & CETESB \\
1- Chr verm/Chr & Mandaville (2002); Fusari \& Fonseca- \\
1- AR de Tubificinae & Guessner (2006) \\
1- AR Exo & Beghelli et al. (2012) \\
\hline \hline
\end{tabular}


Tabela 3 - Limites determinados para cada uma das sete categorias de classificação adotadas para o índice multimétrico da comunidade de macroinvertebrados bentônicos.

\begin{tabular}{ccc}
\hline \hline & Min & Máx \\
\hline Excelente & 0,84 & 0,98 \\
Bom & 0,70 & 0,84 \\
Razoável & 0,56 & 0,70 \\
Impactado & 0,42 & 0,56 \\
Degradado & 0,28 & 0,42 \\
Muito degradado & 0,14 & 0,28 \\
Péssimo & 0,00 & 0,14 \\
\hline \hline
\end{tabular}

Com relação ao índice multimétrico, apenas um ponto, o ponto $A$, pôde ser considerado como em "condições razoáveis", tendo variado nos quatro pontos de 0,41 a 0,58 com média $=0,49$ (impactado) e desvio padrão de 0,07 .

A maior média (menor impacto) foi obtida com o componente espécies exóticas (Exo) presente apenas nas estações $A$ e $B$, enquanto a menor média (maior impacto) foi observado na proporção entre Chironomidae e Tanytarsini. Foi observada maior variação dos componentes relacionados a proporção de organismos tolerantes e relação entre Chironomidae vermelhos e Chironomidae totais indicando que estes componentes são mais sensíveis às diferentes características entre as estações amostrais (tabela 6).

\section{DISCUSSÃO}

Os dados de fósforo e os valores do IET indicam enriquecimento por aporte de fósforo no sistema, sendo mais intenso no ponto $C$. Porém, estes valores elevados de fósforo, contrastam com os baixos valores registrados para clorofila indicando que não são totalmente convertidos em biomassa fitoplanctônica apesar de haver correlação significativa entre ambos.

Tal ocorrência é normal quando há limitação do crescimento fitoplanctônico por outros fatores ambientais como baixa transparência da água. É possível que no caso dos pontos lóticos ( $B$ e $D$ ), a biomassa fitoplanctônica esteja limitada pela luminosidade devido às maiores quantidades de partículas em suspensão em consequência de movimentação mais intensa das águas. Outros fatores como concentrações de nitrogênio, temperatura e interações entre organismos também tendem a alterar esta relação entre fósforo e clorofila a (Carlson, 1977; Lamparelli, 2004; Cunha et al., 2013).

Assim sendo, a CETESB (2013) sugere a avaliação das concentrações de clorofila a e fósforo total quando há divergências entre os respectivos índices. Considerando-se apenas as concentrações de clorofila a, todos os pontos estariam em condições de menor trofia. Porém, ao considerarmos apenas os níveis de fósforo, todos os ambientes estariam na categoria supereutrófico ou acima.

Desse modo, o IET para clorofila permitiria a classificação do corpo d'água em condições de menor trofia que o IET médio ocorrendo o oposto com o IET para fósforo, apesar das variações de ambos estarem diretamente relacionadas.

Isso indica que há potencial para aumento do estado trófico real (clorofila a) que está sendo limitado por outros fatores além do fósforo, podendo o índice para clorofila estar refletindo ainda uma menor trofia de forma artificial caso esteja ocorrendo a aplicação de algicidas no local (CETESB, 2013). Neste sentido recomenda-se, como prevenção, que se adote o índice maior.

Já com relação aos macroinvertebrados, notase que o ponto A encontra-se em melhores condições, composto por uma maior proporção de Tanytarsini,

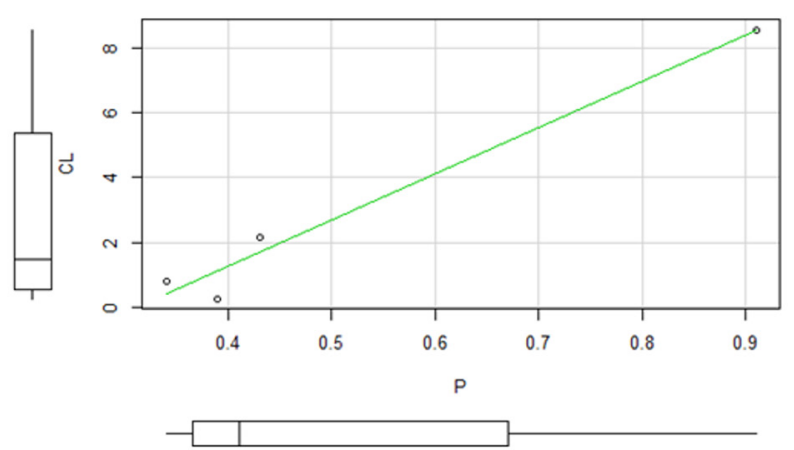

Figura 2 - Relação entre concentrações de clorofila a (CL) e fósforo total (P), p=0,0014. 
Tabela 4 - Valores registrados para clorofila a $(\mathrm{Cl})$; fósforo total $(\mathrm{P})$.

\begin{tabular}{ccc}
\hline \hline Local & $\mathbf{C l}\left(\boldsymbol{\mu g} \cdot \mathrm{L}^{-1}\right)$ & $\mathbf{P}\left(\boldsymbol{\mu g} \cdot \mathbf{L}^{-1}\right)$ \\
\hline $\mathrm{A}$ & 2,14 & 0,43 \\
$\mathrm{~B}$ & 0,27 & 0,39 \\
$\mathrm{C}$ & 8,55 & 0,91 \\
$\mathrm{D}$ & 0,80 & 0,34 \\
\hline \hline
\end{tabular}

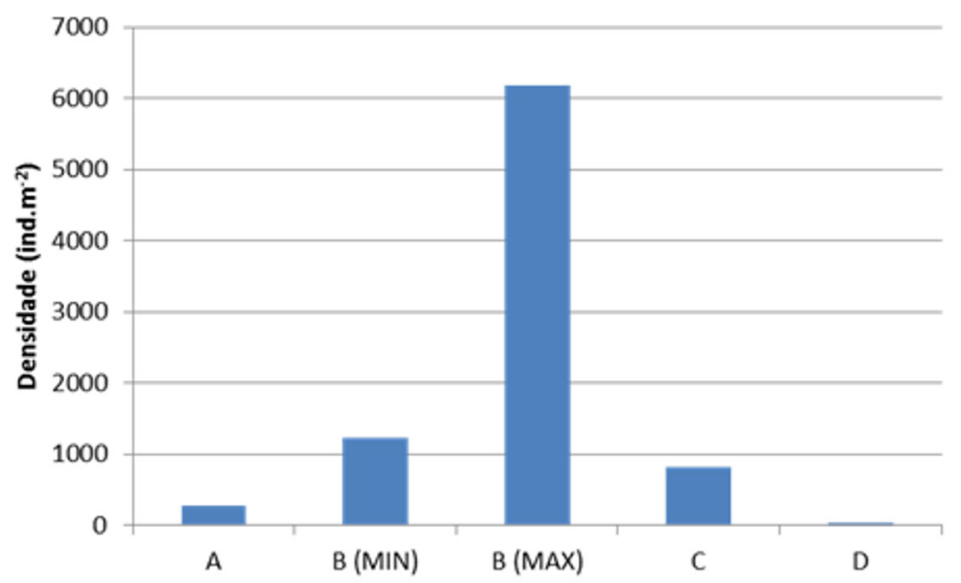

Figura 3 - Densidade de macroinvertebrados bentônicos em diferentes pontos na bacia do rio Jundiaí-Mirim. Valor mínimo registrado para o ponto $\mathrm{B}$, a partir da contagem de 100 indivíduos e valor máximo estimado considerando-se que foram registrados 100 indivíduos em, aproximadamente, um quinto da amostra.

organismos reconhecidos como indicadores de ambientes oligotróficos e mesotróficos (Rosin et al., 2010; Beghelli et al., 2012) e a menor proporção de Chironominae vermelhos que são indicadores de ambientes impactados com pouco oxigênio no fundo (Fusari \& Fonseca-Guessner, 2006). Porém, há a presença da espécie invasora Corbicula fluminea (Müller, 1774) que pode representar uma ameaça biológica à estrutura desta comunidade e do ambiente em questão (Sousa et al., 2008).

Os demais pontos, apesar de apresentarem uma diversidade aparentemente satisfatória na realidade abrigam um predomínio de organismos tolerantes e a ausência ou pequena proporção dos organismos mais sensíveis como os Tanytarsini.

Com relação ao índice multimétrico elaborado, considera-se que o mesmo foi eficiente para diagnóstico da qualidade do ambiente estudado, permitindo a diferenciação das estações amostrais com relação às condições do fundo. Apenas o ponto A foi classificado como em condição "razoável", indicando a presença de impactos no ambiente, mas ainda em intensidades amenas enquanto os demais pontos encontram-se em situações preocupantes refletindo provavelmente, poluição por matéria orgânica, supressão de mata ciliar ou eutrofização do ambiente (Mandaville, 2002; Fusari \& Fonseca-Guessner, 2006; Beghelli et al., 2012; US-EPA, 2012).

Além disso, foram registrados valores muito elevados de densidade no ponto $B$, indicando acúmulo de matéria orgânica que é o alimento da maioria dos macroinvertebrados bentônicos (Beghelli et al., 2012) enquanto no ponto $D$, a densidade esteve anormalmente baixa, provavelmente devido à falta de condições para o estabelecimento destes organismos (sem mata ciliar e em um canal de concreto, com pouco sedimento). Ainda assim, neste último ponto, os únicos táxons presentes são reconhecidamente indicadores de ambientes impactados.

Tabela 5 - IET calculado para diferentes pontos da bacia do rio Jundiaí-Mirim.

\begin{tabular}{ccccc}
\hline \hline & IET CL & IET P & IET médio & Classificação \\
\hline A & 50,46 & 71,21 & 60,83 & Eutrófico \\
B & 38,68 & 66,79 & 52,73 & Mesotrófico \\
C & 57,26 & 75,75 & 66,50 & Supereutrófico \\
D & 48,19 & 66,07 & 57,13 & Mesotrófico \\
\hline \hline
\end{tabular}




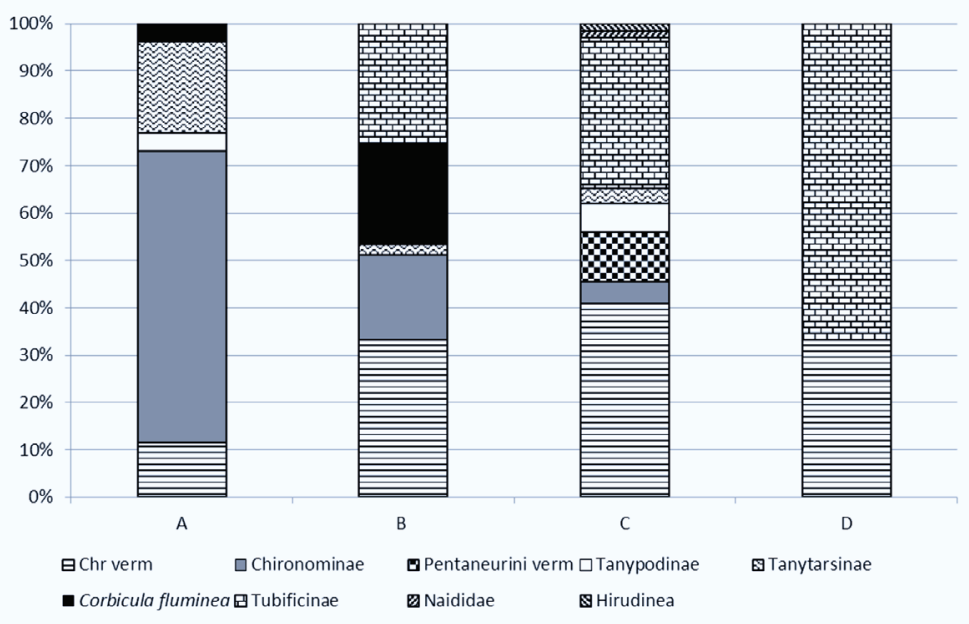

Figura 4 - Composição (abundância relativa) de macroinvertebrados bentônicos da bacia do rio Jundiaí-Mirim conforme os grupos considerados. São considerados grupos associados a condições degradadas: $C$. fluminea; Chironominae vermelhos (Chr verm); Tubificinae e Pentaneurini vermelhos (Pentaneurini verm).

Um importante registro é a presença da espécie exótica $C$. fluminea no ponto $B$. A espécie em questão está entre as invasoras de maior preocupação para ambientes dulcícolas no mundo. Seu sucesso devese, principalmente, às suas características biológicas como crescimento rápido, maturidade sexual precoce, ciclo de vida curto e elevada fecundidade além de sua associação às atividades humanas. Porém não exibe uma ampla faixa de tolerância às alterações ambientais como temperaturas elevadas, hipoxia, baixos $\mathrm{pH}$ ou concentrações de cálcio (Sousa et al., 2008).

Esta espécie invasora pode ainda provocar uma série de prejuízos impactando o ecossistema, levando ao desaparecimento de espécies locais e afetando a cadeia alimentar, ciclos biogeoquímicos como o de fósforo, além de reduzir a transparência da coluna d'água (Zhang et al., 2011).

Neste sentido, observaram-se no ponto $B$ as menores concentrações de clorofila a. Ponto este onde também foi observada elevada proporção de C. fluminea, acima de $20 \%$ da amostra. Estes bivalves são capazes de consumir grandes quantidades de produtores primários realizando filtração a taxas elevadas (Sousa et al., 2008; Descy et al., 2012) o que pode ter contribuído para os valores baixos de clorofila a registrados.

Dentre os prejuízos econômicos, já foi documentado que $C$. fluminea pode ocasionar o entupimento de canais de água, turbinas e sistemas de indústrias que utilizem água bruta bem como sistemas de irrigação (Rosa et al., 2011).

Assim sendo, é proposto o presente índice multimétrico para macroinvertebrados bentônicos como alternativa de baixo custo para diagnóstico expedito sendo ainda necessários estudos futuros em ambientes diferentes para melhor validação do mesmo. Além disso, uma vez que o estudo realizado foi desenhado para uma análise rápida, de diagnóstico, ressalta-se que os dados obtidos estão sujeitos a variações sazonais não contempladas na atual avaliação.

Os resultados indicam que o ambiente em questão encontra-se em situação preocupante conforme indicado tanto pela análise do IET quanto pelos organismos. São sugeridas ações de curto, médio

Tabela 6 - Índices e métricas utilizados para a composição do índice multimétrico calculado. Diversidade de Simpson e índices subtraídos de 1 (valor máximo): family biotic index (Mandaville, 2002); proporção de organismos tolerantes, razão Chironominae-Tanytarsini/ Chironominae+Tanytarsini; Chironominae vermelhos/Chironomidae; proporção de Tubificinae e de espécies exóticas (exo).a

\begin{tabular}{lllllll}
\hline \hline & A & B & C & D & Média & DP \\
\hline Diversidade & 0,57 & 0,75 & 0,71 & 0,44 & 0,62 & 0,14 \\
FBI & 0,37 & 0,23 & 0,17 & 0,07 & 0,21 & 0,13 \\
Tolerantes & 0,85 & 0,20 & 0,17 & 0,00 & 0,31 & 0,37 \\
Chr/Tnt & 0,20 & 0,04 & 0,05 & 0,00 & 0,07 & 0,09 \\
Chr verm/Chr & 0,12 & 0,63 & 0,63 & 1,00 & 0,60 & 0,36 \\
Tubificinae & 1,00 & 0,74 & 0,68 & 0,33 & 0,69 & 0,28 \\
Exo & 0,96 & 0,79 & 1,00 & 1,00 & 0,94 & 0,10 \\
Média & $\mathbf{0 , 5 8}$ & $\mathbf{0 , 4 8}$ & $\mathbf{0 , 4 9}$ & $\mathbf{0 , 4 1}$ & $\mathbf{0 , 4 9}$ & $\mathbf{0 , 0 7}$ \\
Classe & Razoável & Impactado & Impactado & Degradado & Impactado \\
\hline \hline
\end{tabular}


e longos prazos para enfrentamento dos seguintes problemas: supressão de mata ciliar observada em todos os pontos analisados, levantamento de pontos de despejo de efluentes sem tratamento e posterior correção do problema, controle do uso de fertilizantes a base de fósforo na região associado a não ocupação ou, ao menos, a não utilização de tais produtos nas margens de corpos d'água onde deveria estar presente a área de proteção permanente (Brasil, 2012).

Recomendam-se investimentos em pesquisa e monitoramento em relação à espécie exótica $C$. fluminea com o objetivo de se controlar a proliferação da mesma sob risco da região sofrer prejuízos econômicos e ecológicos decorrente da sua proliferação (Pimentel et al., 2005; Sousa et al., 2008). Remoção mecânica, barramento e utilização de substratos artificiais para colonização e remoção são possibilidades de manejo. Além disso, deve-se evitar o transporte dos organismos por meio de embarcações sendo esta uma das principais formas de proliferação da espécie (Mansur et al., 2004; Schmidlin \& Baur, 2007). Sendo fundamental o investimento em pesquisas para melhor conhecimento do comportamento da espécie no Brasil. Uma revisão sobre métodos de controle físicos, químicos e biológicos pode ser obtida em Mansur et al.(2012).

\section{AGRADECIMENTOS}

Os autores agradecem a CAPES e à FAPESP (processos nos 2013/03494-4, 2013/08272-0 e 2012/11890-4) pelo suporte financeiro e ao Instituto Agronômico - IAC pelo apoio.

\section{REFERENCES}

Baron, J.S.; Poff, N.L.; Angermeier, P.L.; Dahm, C.N.; Gleick, P.H.; Hairston, N.G.; Jackson, R.B.; Johnston, C.A.; Richter, B.D. \& Steinman, A.D. 2003. Sustaining Healthy Freshwater Ecosystems. Issues in Ecology. Ecological Society of America, Washington, USA.10: 1-16.

Beghelli, F.G.S.; Dos Santos, A.C.A.; Urso-Guimarães, M.V. \& Calijuri, M.C. 2012. Relationship between space distribution of the benthic macroinvertebrates community and trophic state in a Neotropical reservoir (Itupararanga, Brazil). Biota Neotropica.12(4): 114-124.

Brasil. 2012. Presidência da República, Casa Civil. Lei n. 12651 de 25 de maio de 2012.

Caldas, J. S. \& Sanches Filho, P. 2013. Determinação de $\mathrm{Cu}, \mathrm{Pb}$ e $\mathrm{Zn}$ na região do Pontal da Barra,
Laranjal (Laguna dos Patos, Pelostas, RS, Brasil). Braz. J. Aquat. Sci. Technol.17(1): 13-18.

Carlson, R. E. 1977. A trophic state index for lakes. Limnol. and Oceanogr. 2(2): 361-369.

Companhia Ambiental do Estado de São Paulo - CETESB. 2013. Índice de estado trófico. Disponível em: <http://www.cetesb.sp.gov.br/ userfiles/file/agua/aguas-superficiais/aguasinteriores/documentos/indices/04.pdf>. Acessado em 03/11/2013.

Companhia Ambiental do Estado de São Paulo CETESB. 2014. ICB - Índice da comunidade bentônica. In: Índice de qualidade das águas. Disponível em: <http://www.cetesb.sp.gov.br/ userfiles/file/agua/aguas-superficiais/aguasinteriores/documentos/indices/07.pdf>. Acessado em 21/07/2014

Costigan, K.H. \& Daniels, M.D. 2012. Damming the prairie: Human alteration of Great Plains river regimes. Journal of Hydrology. 444: 90-99.

Cunha, D.G.F.; Grull, D.; Damato, M.; Blum, J.R.C.; Sergio, E.; Lutti, J.E.I. \& Mancuso, P.C.S. (2011). Contiguous urban rivers should not be necessarily submitted to the same management plan: the case of Tietê and Pinheiros Rivers (São Paulo-Brazil). Anais da Academia Brasileira de Ciências, 83(4): 1465-1480.

Cunha, D.G.F.; Calijuri, M.C. \& Lamparelli, M.C. (2013). A trophic state index for tropical/subtropical reservoirs (TSItsr). Ecological Engineering. 60: $126-134$.

Descy, J.P.; Leitao, M.; Everbecq, E.; Smitz, J.S. \& Delie 'Ge, J.F.O. 2012. phytoplankton of the River Loire, France: a biodiversity and modelling study. Journal of plankton research. 34(2): 120-135.

Dosskey, M. G., Vidon, P., Gurwick, N. P., Allan, C. J., Duval, T. P. \& Lowrance, R. 2010. The role of riparian vegetation in protecting and improving chemical water quality in streams. JAWRA. 46: 261-277.

Floehr, T.; Xiao, H.; Scholz-Starke, B.; Wu, L.; Hou, J.; Yin, D.; Zhang, X; Ji, R.; Yuan, X.; Ottermanns, R.; Roß-Nickoll, M.; Schäffer, A. \& Hollert, H. 2013. Solution by dilution?-A review on the pollution status of the Yangtze River. Environ Sci Pollut Res. 20: 6934-6971.

Freitas, E.P. 2012. Análise integrada do mapa de uso e ocupação das terras da microbacia do rio Jundiaí-Mirim para fins de gestão ambiental. Dissertação de Mestrado - Instituto Agronômico IAC. Campinas, 120p.

Fundação Sistema Estadual de Análise de Dados (SEADE). 2014. PIB Municipal 2011. Disponível em: https://www.seade.gov.br/produtos/pibmun/ index.php. Acessado em 31/03/2014. 
Fusari, L.M. \& Fonseca-Gessner, A.A. 2006. Environmental assessment of two small reservoirs in southeastern Brazil, using macroinvertebrate community metrics. Acta Limnol. Bras.18(1): 89-99.

Hilsenhoff, W.L. 1988. Rapid field assessment of organic pollution with a family-level biotic index. J. N. Am. Benthol. Soc., 7(1):65-68.

Instituto Brasileiro de Geografia e Estatística - IBGE. 2010. Contas regionais do Brasil, 2010. Disponível em: < http://www.ibge.gov.br/home/estatistica/ economia/contasregionais/2010/default.shtm> Acessado em: 18/11/2013.

Knowler, D. 2005. Reassessing the costs of biological invasion: Mnemiopsis leidyi in the Black sea. Ecological Economics, 52 (2): 187-199.

Lamparelli, M.C. 2004. Grau de trofia em corpos d'água do Estado de São Paulo: avaliação dos métodos de monitoramento. Tese de doutorado. Instituto de Biociências da Universidade de São Paulo. 238p.

Lawal, M. O.; Samuel, O. B.; Mogekwu, T. O. \& Bolaji, D. A. 2013. Toxicity of two households liquid soaps on Poecilia reticulata Peters, 1859. Braz. J. Aquat. Sci. Technol.17(1): 35-41.

Leal, J.H. 2013. Bivalves. Bailey-Matthews Shell Museum, Florida, USA. Disponível em: <http:// www.scvz.org/zoolcv/vol1no1/Lopes $\% 20$ CV\%20 bivalves.pdf>. Acessado em 01/11/2013.

Li, L.; Zheng, B. \& Liu, L. 2010. Biomonitoring and Bioindicators Used for River Ecosystems: Definitions, Approaches and Trends. Procedia Environmental Sciences. 2: 1510-1524.

Mandaville, S.M. 2002. Benthic macroinvertebrates in freshwaters - taxa tolerance values, metrics and protocols. (Project H-1) Soil; water conservation society of metro Halifax. 128p.

Mansur, M.C.D.; Callil, C.T.; Cardoso, F.R. \& Ibarra, J.A.A. 2004. Uma retrospectiva e mapeamento da invasão de espécies de Corbicula (Mollusca, Bivalvia, Veneroida, Corbiculidae) oriundas do Sudeste asiático na América do Sul. In: Silva \& Souza (org.). Água de lastro e bioinvasão, Rio de Janeiro, RJ: Editora Interciência, 39-58 pp.

Mansur, M.C.D.; dos Santos, C.P.; Pereira, D.; Paz, I.C.P.P.; Zurita, M.L.L.; Rodriguez, M.T.R.; Nehrke, M.V. \& Bergonci, P.E.A. 2012. Moluscos límnicos invasores no Brasil : biologia, prevenção e controle. $1^{a}$ Edição. Redes Editora, Porto Alegre, 412 p.

Marchese, M. R. 2009. Annelida. Oligochaeta. In: Dominguéz, E.; Fernández, H. R. (coord.). Macroinvertebrados bentónicos sudamericanos. Sistemática y biologia. Fundación Miguel Lillo, Tucumán, Argentina. 551-565 pp.

Medeiros, G.A.; Tresmondi, A.C.C.; Queiroz, B.P.V.; Melo, C.A.; Rosa, A.H.; Negro, C.V.; Fraceto,
L.F. \& Ribeiro, A.I. 2013. Evaluation of metals in water and sediments of micro-basins in the city of Americana, São Paulo state, Brazil. WIT Transactions on Ecology and the Environment, 172:201.

Melo, C.E.; Machado, F.A. \& Pinto-Silva, V. 2004. Feeding habits of fish from a stream in the savanna of Central Brazil, Araguaia Basin. Neotrop. ichthyol. 2(1): 37-44.

Monteagudo, L.; Moreno, J.L. \& Picazo, F. 2012. River eutrophication: Irrigated vs. non-irrigated agriculture through different spatial scales. Water Research.46(8): 2759-2771.

Moraes, J.F.L. (coord.). 2003. Diagnóstico agroambiental para gestão e monitoramento da bacia do rio Jundiaí-Mirim. Relatório Final $-2^{a}$ Fase. Processo Fapesp: 98/14181-5: Execução: Instituto Agronômico de Campinas - IAC, Instituto de Economia Agrícola - IEA, Prefeitura do Município de Jundiaí, DAE S/A. Campinas - SP.

Moreira, J.C. Peres, F.; Simões, A.C.; Pignati, W.A.; Dores, E.C.; Vieira, S.N.; Strüssmann, C. \& Mott, T. 2012. Contaminação de águas superficiais e de chuva por agrotóxicos em uma região do estado do Mato Grosso. Ciênc. saúde coletiva. 17(6): 1557-1568.

Odum, E.P. 1988. Ecologia. Rio de Janeiro, RJ: Ed. Guanabara Koogan S. A. 434p.

Organização das Nações Unidas (ONU), 1992. Proteção da qualidade e do abastecimento dos recursos hídricos: Aplicação de critérios integrados no desenvolvimento, manejo e uso dos recursos hídricos. In:Conferência das Nações Unidas Sobre Meio Ambiente e Desenvolvimento., Rio de Janeiro, RJ, Brasil, seção II, cap. 18.

Pedrazzi, F.J.M.; Conceição, F. T.; Sardinha, D. S., Moschini-Carlos, V. \& Pompêo, M. 2013. Spatial and Temporal Quality of Water in the Itupararanga Reservoir, Alto Sorocaba Basin (SP), Brazil. Journal of Water Resource and Protection, 5: 64-71.

Pimentel, D.; Zuniga, R. \& Morrison, D. (2005). Update on the environmental and economic costs associated with alien-invasive species in the United States, Ecological Economics. 52: 273-288.

Rodgher, S.; Espíndola, E.L.G.; Rocha, O; Fracácio, R; Pereira, R.H.G. \& Rodrigues, M.H.S. 2005. Limnological and ecotoxicological studies in the cascade of reservoirs in the Tietê River (São Paulo, Brazil). Brazilian Journal of Biology. 65(4): 697-710.

Rosa, I.C.; Pereira, J.L.; Gomes, J.; Saraiva, P.M.; Gonçalves, F. \& Costa, R. 2011. The Asian clam Corbicula fluminea in the European freshwaterdependent industry: A latent threat or a friendly enemy? Ecological Economics. 70: 1805-1813. 
Rosin, G.C.; Mangarotti, D.P.O. \& Takeda, A.M. 2010. Chironomidae (Diptera) community structure in two subsystems with different states of conservation in a floodplain of southern Brazil. Acta Limnologica Brasiliensia. 22(3): 276-286.

Schmidlin, S. \& Baur, B. 2007. Distribution and substrate preference of the invasive clam Corbicula fluminea in the river Rhine in the region of Basel (Switzerland, Germany, France). Aquatic Sciences. 69(1): 153-161.

Smith, A.P.; Western, A.W.; Hannah, M.C. 2013. Linking water quality trends with land use intensification in dairy farming catchments. Journal of Hydrology. 476: 1-12.

Sousa, R.; Nogueira, A.J.A.; Gaspar, M.B.; Antunes, C. \& Guilhermino, L. 2008. Growth and extremely high production of the non-indigenous invasive species Corbicula fluminea (Müller, 1774): Possible implications for ecosystem functioning. Estuarine Coastal and Shelf Science. 80(2): 289-295.

Stewart, A.J ; Smith, J.G. \& Loar, J.M. 2011. LongTerm Water-Quality Changes in East Fork Poplar Creek, Tennessee: Background, Trends, and Potential Biological Consequences. Environmental Management. 47(6): 1021-1032.

Thevenon, F.; Graham, N.D.; Herbez, A.; Wildi, W. \& Poté, J. 2011. Spatio-temporal distribution of organic and inorganic pollutants from Lake Geneva (Switzerland) reveals strong interacting effects of sewage treatment plant and eutrophication on microbial abundance. Chemosphere. 84(5): 609-617.
Trivinho-Strixino, S. 2011. Larvas de Chironomidae: guia de identificação. São Carlos, SP: gráfica da Universidade Federal de São Carlos, 371p.

Tundisi, J.G. \& Matsumura-Tundisi, T.2008. Limnologia. São Paulo, SP: Oficina de Textos. 632p.

US Environmental Protection Agency - US - EPA. 2003. Monitoring and assessment program. Assessment and Watershed Protection Division Office of Wetlands, Oceans and Watershed. 24p.

US Environmental Protection Agency - US - EPA. 2012. Rapid Biological Assessment Protocols: An Introduction. Watershed Academy Web. 34p.

Wetzel, R. G. \& Likens, G. E. 1991. Limnological analysis. 2 ed. New York, W. B. Saunders Company. $391 \mathrm{p}$.

Zanata, L.H.; Espindola, E.L.G.; Rocha, O. \& Pereira, R.H.G. 2008. Morphological abnormalities in Cladocera (Branchiopoda) in a cascade of reservoirs in the middle and lower Tietê river (São Paulo, Brazil). Brazilian Journal of Biology. 68(3): 681-682.

Zhang, L.; Shen, Q.; Hu, H.; Shao, S. \& Fan, C. 2011. Impacts of Corbicula fluminea on Oxygen Uptake and Nutrient Fluxes across the Sediment-Water Interface. Water, Air, \& Soil Pollution. 220(1-4): p 399-411. 